\title{
SHORT REPORT \\ Tuberculosis and risk of acute myocardial infarction: a propensity score-matched analysis
}

\author{
M. A. HUAMAN ${ }^{1,2}$, R. J. KRYSCIO ${ }^{3}$, C. J. FICHTENBAUM ${ }^{1}$, D. HENSON ${ }^{2}$, \\ E. SALT ${ }^{4}$, T. R. STERLING ${ }^{5}$ AND B. A. GARVY ${ }^{2,6}$ \\ ${ }^{1}$ Department of Internal Medicine, University of Cincinnati College of Medicine, Cincinnati, OH, USA \\ ${ }^{2}$ Department of Medicine, University of Kentucky College of Medicine, Lexington, KY, USA \\ ${ }^{3}$ Departments of Biostatistics and Statistics, University of Kentucky College of Public Health, Lexington, KY, USA \\ ${ }^{4}$ University of Kentucky College of Nursing, Lexington, KY, USA \\ ${ }^{5}$ Department of Medicine, Vanderbilt University School of Medicine, Nashville, TN, USA \\ ${ }^{6}$ Department of Microbiology, Immunology and Molecular Genetics, University of Kentucky College of \\ Medicine, Lexington, KY, USA
}

Received 4 October 2016; Final revision 13 January 2017; Accepted 19 January 2017; first published online 16 February 2017

\section{SUMMARY}

Several pathogens have been associated with increased cardiovascular disease (CVD) risk. Whether this occurs with Mycobacterium tuberculosis infection is unclear. We assessed if tuberculosis disease increased the risk of acute myocardial infarction (AMI). We identified patients with tuberculosis index claims from a large de-identified database of $\sim 15$ million adults enrolled in a U.S. commercial insurance policy between 2008 and 2010. Tuberculosis patients were 1:1 matched to patients without tuberculosis claims using propensity scores. We compared the occurrence of index AMI claims between the tuberculosis and non-tuberculosis cohorts using Kaplan-Meier curves and Cox Proportional Hazard models. Data on 2026 patients with tuberculosis and 2026 propensity-matched patients without tuberculosis were included. AMI was more frequent in the tuberculosis cohort compared with the non-tuberculosis cohort, $67(3.3 \%)$ vs. $32(1 \cdot 6 \%)$ AMI cases, respectively, $P<0 \cdot 01$. Tuberculosis was associated with an increased risk of AMI (adjusted hazard ratio (HR) 1.98, 95\% confidence intervals (CI) $1 \cdot 3-3 \cdot 0$ ). The results were similar when the analysis was restricted to pulmonary tuberculosis (adjusted HR $2 \cdot 43,95 \%$ CI 1.5-4·1). Tuberculosis was associated with an increased risk of AMI. CVD risk assessment should be considered in tuberculosis patients. Mechanistic studies of tuberculosis and CVD are warranted.

Key words: Acute myocardial infarction, cardiovascular disease, risk, tuberculosis.

\section{INTRODUCTION}

The burden of tuberculosis and cardiovascular disease (CVD) is enormous worldwide [1, 2]. Tuberculosis

\footnotetext{
* Author for correspondence: M. A. Huaman, Division of Infectious Diseases, University of Cincinnati, 231 Albert Sabin Way, Cincinnati, OH 45267, USA.

(Email: moises.huaman@uc.edu)
}

and CVD may not only occur concomitantly, but also affect the risk of one another. Potential contributors to CVD risk in tuberculosis may include a direct effect of Mycobacterium tuberculosis on the myocardium and coronary arteries, increased immune activation and cytokine production, and cross-reactivity of anti-mycobacterial antibodies against cardiovascular tissue [3]. 
We used a registry of $\sim 15$ million persons enrolled in a U.S. commercial insurance policy representing 1284681 prescribers and 3631 health care provider designations from all geographic U.S. regions [4], to determine whether tuberculosis increased the risk of acute myocardial infarction (AMI) after adjusting for traditional CVD risk factors.

\section{METHODS}

We identified patients with and without an index tuberculosis disease claim (International Classification of Diseases, ninth revision (ICD-9) codes, 010.0-018.9) between 2008 and 2010. We included patients aged 25 years or older who had complete administrative data. Younger patients were excluded due to much lesser likelihood of AMI occurrences. We excluded patients with human immunodeficiency virus (HIV)/ AIDS (acquired immune deficiency syndrome) claims (ICD-9 code, 042) and organ or tissue transplantation claims (ICD-9 codes, V42.0-V42.9) as both tuberculosis and AMI could manifest very differently in these populations. Tuberculosis patients were required not to have a tuberculosis claim the year prior, to improve likelihood of selection for new tuberculosis diagnoses within the study period. Patients with claims related to latent tuberculosis infection (ICD-9 codes, 795.5, 795.51, 795.52) and/or non-tuberculous mycobacterial infections (ICD-9 codes, 030.0-031.9) were excluded from the study.

We then identified patients without tuberculosis claims to establish a comparison cohort. We used propensity scores for 1:1 matching of tuberculosis and non-tuberculosis patients based on the nearest neighbor method with a caliper width set at $0 \cdot 2$ [5]. To calculate propensity scores, we included candidate risk factors for both tuberculosis and AMI. Thus, propensity scores were created through a logistic regression for the predicted probability of having a tuberculosis claim as a function of birth year, sex, race, hypertension (ICD-9 codes, 401.0-401.9), diabetes (ICD-9 codes, 250.0-250.9), hyperlipidemia (ICD-9 codes, 272.0-272.9), obesity (ICD-9 codes, 270.0-270.9), tobacco use (ICD-9 code, 305.1), chronic kidney disease (ICD-9 code, 585.9) and major autoimmune condition which included ICD-9 codes for systemic lupus erythematosus $(710 \cdot 0)$, rheumatoid arthritis and other inflammatory polyarthropathies (714.0-714.9), psoriasis (696.0-696.8), Crohn's disease (555.0-555.9), and ulcerative colitis $(556 \cdot 0-556 \cdot 9)$.
We set the date of index tuberculosis claim as the start date for each tuberculosis and non-tuberculosismatched pair, and looked for the occurrence of index AMI claims (ICD-9 codes, 410·0-410.9) within the following year period. Thus patients were followed through to the date of index AMI claim, death, insurance disenrollment or completion of 1-year period, whichever happened first. Dates available in the study database included information on month and year therefore we rounded all dates to the first day of their corresponding month and year. To better assess the timing of index AMI claim in relation to the index tuberculosis claim, we excluded those pairs where an AMI index claim was first reported within the same month of the index tuberculosis claim. Patients with an index AMI claim were required not to have an AMI claim the year prior to their follow-up start date, to improve likelihood of selection for new AMI diagnoses within the study period.

Baseline characteristics of the matched cohorts were compared using $\chi^{2}$ tests to ensure there were no residual significant differences. We used the KaplanMeier method to estimate the proportion of patients free of AMI over time. The log-rank test was used to compare differences in AMI between cohorts. Cox Proportional Hazards models for paired data were used to assess tuberculosis as a risk factor for AMI after adjusting for the other risk factors. The results of Cox models were presented as hazard ratios (HR) accompanied by $95 \%$ confidence intervals (CI). All analyses were performed using Stata software (version 12.0; StataCorp, Texas, USA) with $P<0.05$ as the level of statistical significance. This project was exempt of institutional review board review as it involved the analysis of available, existing, de-identified data.

\section{RESULTS}

Data on 2026 patients with tuberculosis and 2026 propensity score-matched patients without tuberculosis were included in the study. There were no significant differences between the tuberculosis and non-tuberculosis cohorts in terms of age $\geqslant 65$ years $(56.9 \%$ vs. $57 \cdot 3 \%, P=0.78)$, male sex $(43.7 \%$ vs. $43 \cdot 5 \%, P=0 \cdot 89)$, black race $(17 \cdot 7 \%$ vs. $17 \cdot 3 \%, P=$ $0 \cdot 74)$; Hispanic ethnicity ( $3.9 \%$ vs. $4 \cdot 2 \%, P=0.63)$, diabetes mellitus $(50 \cdot 3 \%$ vs. $50 \cdot 5 \%, P=0 \cdot 88)$, hypertension (65.3\% vs. $64.7 \%, P=0.72)$, hyperlipidemia $(56 \cdot 3 \%$ vs. $55 \cdot 9 \%, P=0.83)$, obesity $(16 \cdot 2 \%$ vs. $15 \cdot 2 \%, P=0 \cdot 34)$, tobacco use $(17 \cdot 3 \%$ vs. $17 \cdot 8 \%, P=0 \cdot 65)$, chronic kidney 


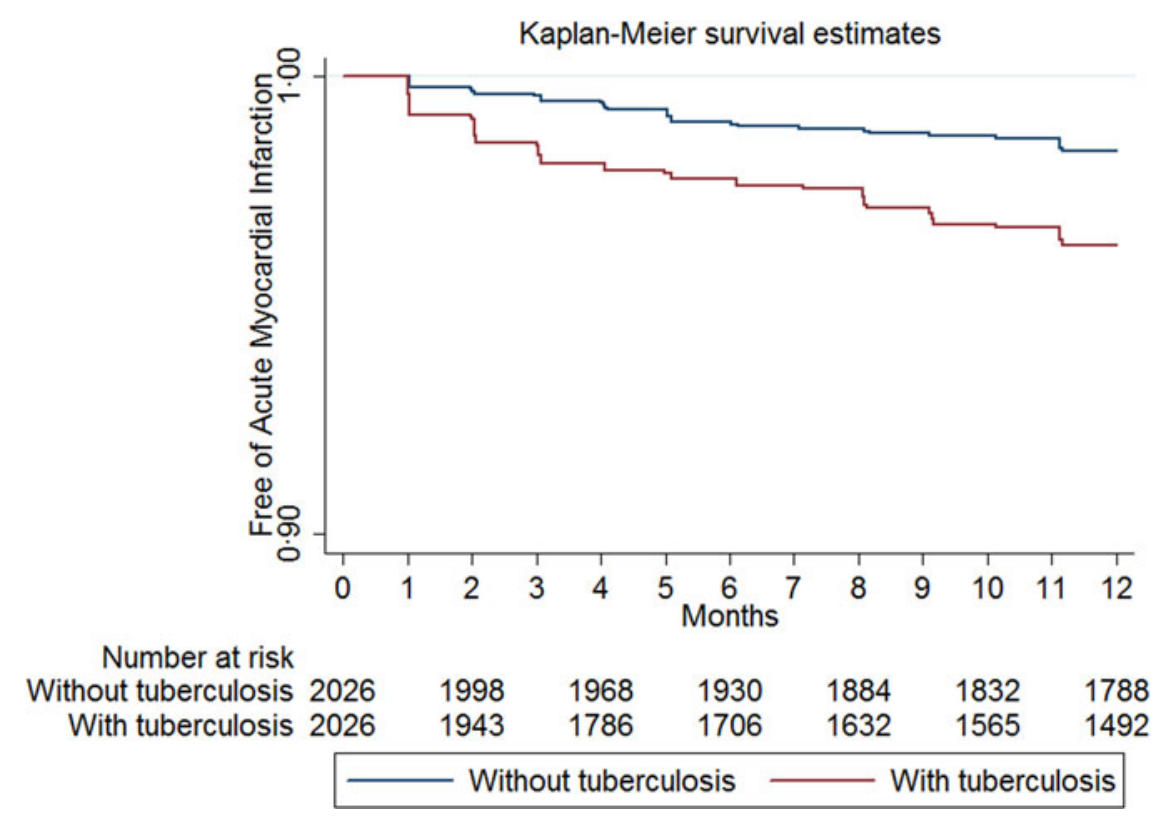

Fig. 1. Kaplan-Meier curves for acute myocardial infarction in the study groups.

disease $(16 \cdot 9 \%$ vs. $16 \cdot 7 \%, P=0.87)$, or major autoimmune disease $(19 \cdot 8 \% v s .19 \cdot 6 \%, P=0 \cdot 87)$, indicating sufficient propensity score matching.

There were more cases of AMI in the tuberculosis cohort (67 cases, 3.3\%) than the non-tuberculosis cohort (32 cases, $1 \cdot 6 \%$ ), $P<0.01$. The KaplanMeier survival curves are shown in Figure 1 (log-rank test, $P<0.01)$. The risk of developing AMI in patients with tuberculosis claims was higher compared with patients without tuberculosis claims and similar baseline characteristics and comorbidities (adjusted HR $1 \cdot 98,95 \%$ CI $1 \cdot 3-3 \cdot 0$ ). The proportional hazards assumption was not violated based on the Schoenfeld residuals test, $P=0 \cdot 25$. The results were similar when the analysis was restricted to pulmonary tuberculosis claims (adjusted HR 2.43, 95\% CI $1 \cdot 5-4 \cdot 1$ ). Hypertension (adjusted HR 1.91, 95\% CI 1.1-3.6), chronic kidney disease (adjusted HR 2.68, 95\% CI $1 \cdot 7-4 \cdot 2$ ), and tobacco use (adjusted HR $1 \cdot 6,95 \%$ CI 1.1-2.6) were independently associated with AMI.

\section{DISCUSSION}

In this propensity score-matched analysis of commercially insured U.S. patients with multiple comorbidities, we showed that tuberculosis disease was associated with an approximate twofold increased risk of AMI by 1 year after a tuberculosis disease index claim. Similar results were obtained when the analysis was restricted to pulmonary tuberculosis.
Our findings are consistent with a large populationbased retrospective cohort study conducted in Taiwan which showed a 1.4-fold increased subsequent risk of acute coronary syndrome (ACS), a composite outcome that includes unstable angina and AMI diagnoses, among patients with a history of tuberculosis disease [6]. The Taiwan study showed higher incidence rate ratios of ACS between tuberculosis and nontuberculosis patients when comorbidities such as hypertension, diabetes, or hyperlipidemia were present. Therefore, we reason that our higher risk estimate may be explained by the overall higher frequency of traditional CVD risk factors in our population as compared with the Taiwan study. In addition, the Taiwan study looked at ACS, whereas we focused our analysis on AMI only. Notably, a similar analysis of the Taiwan national registry showed a $1 \cdot 5$-fold increased risk of ischemic stroke among persons with a history of tuberculosis [7], which indicates that the effect of tuberculosis in atherosclerotic disease goes beyond coronary heart disease. A major strength of our study was the use of propensity score matching to control for demographics and available information on relevant comorbidities among patients with and without tuberculosis, allowing a more accurate analysis of the relationship between tuberculosis and AMI.

Numerous intracellular pathogens that establish chronic and/or latent infection have been implicated in the development of CVD; i.e. HIV, cytomegalovirus, and Chlamydia pneumoniae [8]. The connection between infection and CVD centers on several 
mechanisms including direct cellular infection and damage, increased immune activation, or induction of autoimmunity [9]. Atherogenesis and plaque rupture are, in part, dependent on the role of activated monocytes/macrophages and Th1 effector cells releasing interferon- $\gamma$, interleukin-1, and other cytokines [10]. Patients with tuberculosis share this pro-inflammatory profile of activated monocyte/macrophages, $T$ cells and elevated inflammatory cytokines [3]. Tuberculosis may also induce autoimmunity through molecular mimicry of the heat shock protein (HSP) system. Antibodies to mycobacterial HSP65 may cross-react with human HSP60, as studies indicate that antibodies to HSP65 correlate with the development of atherosclerosis [11]. Cases of sudden cardiac death and arteritis suggested a direct effect of $M$. tuberculosis on the myocardium and coronary arteries but these seem to be rare occurrences [12].

There are a number of factors that may affect the conclusions of this study. We could not obtain detailed information on the length of time or severity of comorbidities such as hypertension, diabetes, renal disease, or autoimmune disorders, which could affect the risk of AMI differently. Quantification of tobacco use or body mass index for each patient was not available. However, we captured diagnostic codes for tobacco use and obesity as proxies. This database has been used for analyses of fungal and mycobacterial infections including tuberculosis showing patterns consistent with what has been reported in the literature [4]. However, because tuberculosis is managed at local U.S. health departments, we could not further confirm tuberculosis diagnoses or access antituberculosis treatment data for our study population. Most tuberculosis patients included in the study would be expected to be taking anti-tuberculosis medications within a year of their first tuberculosis insurance claim. This could be of importance, as rifamycin-containing regimens could decrease the effectiveness of drugs used to treat traditional CVD risk factors such as hypertension, diabetes and hyperlipidemia, and thereby increase the risk of AMI. The incidence of AMI in the non-tuberculosis group $(1 \cdot 6 \%)$ was $1 \cdot 5-2$ times higher than the expected incidence of AMI in a U.S. population with similar age and sex distribution [13]. This suggests that some of the AMI events extracted from the study registry could have been prevalent cases; or that our study population was at an overall higher risk for CVD than the general population, as suggested by the presence of multiple comorbidities in our population. As with all administrative databases there may be significant biases and/or errors in coding and reporting though we have no reason to suspect they would be channeled in one direction or another. Finally, it is important to mention that our results are not necessarily generalizable to uninsured populations or settings where tuberculosis is highly endemic. Since the prevalence of CVD and CVD risk factors is rising globally, research is greatly needed to determine whether tuberculosis would have a similar effect over AMI in populations that are traditionally more vulnerable to tuberculosis (i.e. homeless individuals, intravenous drug users, alcohol users, persons who live in poverty and resource-constraint settings).

In conclusion, tuberculosis was associated with an approximate twofold increased risk of AMI in this commercially insured U.S. population with multiple comorbidities. These findings suggest that CVD assessment should be considered in tuberculosis patients. Our results need further validation in longitudinal studies that control for CVD risk factors in greater detail. Further mechanistic studies of tuberculosis and CVD are warranted.

\section{ACKNOWLEDGEMENTS}

C.J.F. has received research support to the University of Cincinnati from Gilead, Pfizer, BMS, ViiV, Janssen, and Merck. This research was supported in part by the National Center for Research Resources and the National Center for Advancing Translational Sciences, National Institutes of Health (NIH), through Grant UL1TR000117 (MAH). The authors would like to thank Darren Henderson, database administrator at the University of Kentucky Center for Clinical and Translational Science for extracting and providing the data for this project.

\section{DECLARATION OF INTEREST}

None.

\section{REFERENCES}

1. World Health Organization. Global Status Report on Nonconcommunicable Diseases 2014. Geneva, Switzerland: WHO, 2014.

2. World Health Organization. Global Tuberculosis Report 2015. Geneva, Switzerland: WHO, 2015.

3. Huaman MA, et al. Tuberculosis and cardiovascular disease: linking the epidemics. Tropical Diseases, Travel Medicine and Vaccines 2015; 1: 10, 1-7. 
4. Salt E, et al. Risk factors for targeted fungal and mycobacterial infections in patients taking tumor necrosis factor inhibitors. Arthritis \& Rheumatology 2016; 68: 597-603.

5. Austin PC. Optimal caliper widths for propensity-score matching when estimating differences in means and differences in proportions in observational studies. Pharmaceutical Statistics 2011; 10: 150-161.

6. Chung WS, et al. Tuberculosis increases the subsequent risk of acute coronary syndrome: a nationwide population-based cohort study. International Journal of Tuberculosis and Lung Disease 2014; 18: 79-83.

7. Sheu JJ, et al. Tuberculosis and the risk of ischemic stroke: a 3-year follow-up study. Stroke 2010; 41: 244-249.

8. Rosenfeld ME, Campbell LA. Pathogens and atherosclerosis: update on the potential contribution of multiple infectious organisms to the pathogenesis of atherosclerosis. Thrombosis and Haemostasis 2011; 106: 858-867.

9. Epstein SE, et al. Insights into the role of infection in atherogenesis and in plaque rupture. Circulation 2009; 119: $3133-3141$.

10. Hansson GK. Inflammation, atherosclerosis and coronary artery disease. New England Journal of Medicine 2005; 352: 1685-1695.

11. Xu Q, et al. Association of serum antibody to heatshock protein 65 with carotid atherosclerosis. Lancet 1993; 341: 255-259.

12. Liu A, Hu Y, Coates A. Sudden cardiac death and tuberculosis - how much do we know? Tuberculosis (Edinb) 2012; 92: 307-313.

13. Mozaffarian D, et al. Heart disease and stroke statistics-2016 update: a report from the American Heart Association. Circulation 2016; 133: e38-e360. 